\title{
Success of dupilumab as a monotherapy in an adult patient affected by severe uncontrolled asthma and atopic dermatitis
}

\section{Dear Editor,}

Atopic dermatitis and asthma are chronic Th2-driven inflammatory diseases; an important role in their pathogenesis is played by IL-4 and IL-13. Dupilumab is a fully human monoclonal antibody against the $\alpha$ subunit of IL-4 and IL-4/IL-13 receptor complexes inhibiting both IL-4 and IL-13 pathways. ${ }^{1}$ It is indicated for the treatment of difficult-tothreat atopic dermatitis in adults and as an add-on maintenance therapy in moderate-to-severe asthma with a type 2 inflammation, characterized by eosinophilia, and/or elevated fractional exhaled nitric oxide, inadequately controlled with high dose inhaled corticosteroids plus another medicinal product. $^{2}$

In 2019 a 35 years old Caucasian woman presented to our attention complaining about severe persistent atopic dermatitis, previously treated unsuccessfully with: clobetasol dipropionate $0.05 \%$ and tacrolimus $0.1 \%$ ointments, systemic corticosteroids, cyclosporine (200 mg/die), and mycophenolate mofetil. In anamnesis asthma with high IgE levels (15.000 KU/L) diagnosed in October 2008: spirometry revealed percentage of predicted Forced Expiratory Volume in the first second (FEV1) value 33\% while the FEV1/Forced Vital Capacity (FVC) ratio was $40 \%$, indicative for a severe obstructive pattern. Thus, oxygen therapy was instituted (18 hours/day). Omalizumab
$(150 \mathrm{mg} / 2 \mathrm{w}$ ) therapy, preceded by plasmaferesis because of high IgE levels, resulted in a lung function improvement without proper control of atopic dermatitis.

Widespread erythematous plaques with erosions and crusted lesions involving skin of the face, neck, arms, legs, and hands were observed at the clinical examination. Flexural areas showed lichenification and excoriations, due to continuous scratching (Figure 1A). The patient complained of constant and intense pruritus and poor quality of life. Severity indexes showed: Eczema Area and Severity Index (EASI) 40, Dermatology Life Quality Index (DLQI) 28, Numerical Rating Scale (NRS) itch 10, and NRS sleep 10.

Therefore, dupilumab (600 mg then $300 \mathrm{mg} / 2 \mathrm{w}$, s.c.) therapy was started in place of omalizumab.

Skin improvement was quickly achieved. After 4 weeks of therapy: EASI 25, DLQI 15, NRS itch 4, and NRS sleep 4 (Figure 1B). At week 16: EASI 10, DLQI 5, NRS itch 2, and NRS sleep 2 (Figure 1C).

Spirometry was repeated before treatment with dupilumab: percentage of predicted FEV1 value was $41 \%$ while the FEV1/FVC ratio was $50 \%$. Sixteen weeks later these values were considerably improved: percentage of predicted FEV1 value was $61 \%$, while the FEV1/FVC ratio was 74\%. Moreover respiratory difficulties improved:
(A)

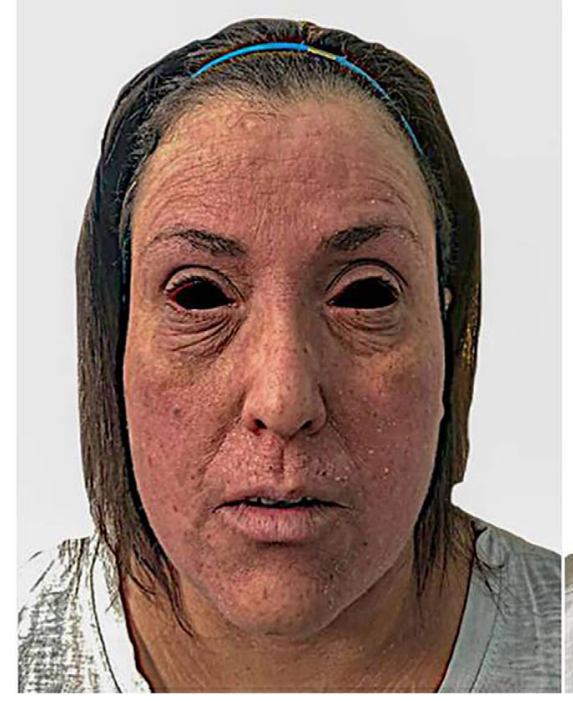

(B)

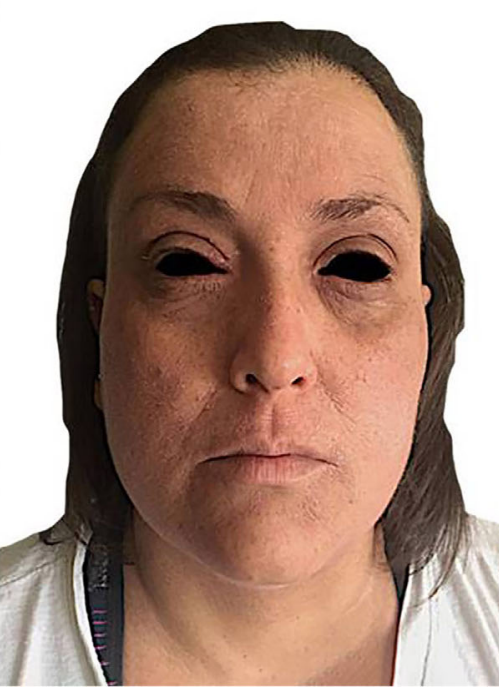

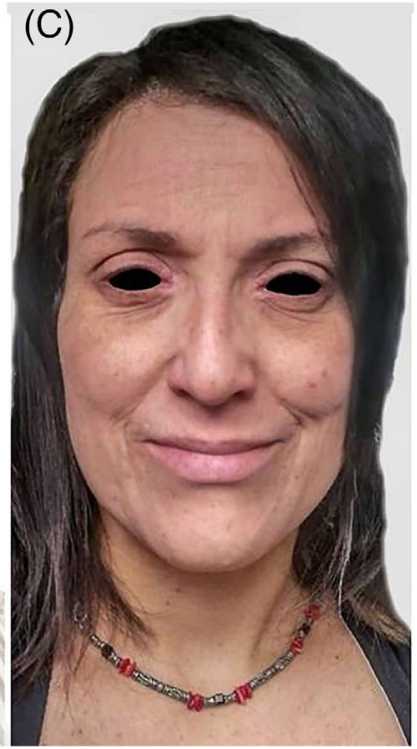

FIGURE 1 A, Patient's clinical condition before starting dupilumab. B, Patient's clinical condition after 4 weeks of therapy with dupilumab. C, Patient's clinical condition after 16 weeks of therapy with dupilumab 
before starting dupilumab diurnal symptoms (tightness in the chest, coughing, and wheezing) were almost daily and nocturnal awakenings were frequent, after 16 weeks diurnal symptoms became infrequent and nocturnal awakenings ceased.

Currently IgE levels have dropped to $404 \mathrm{KU} / \mathrm{L}$, with almost complete resolution of eczematous lesions, lung function improvement, and absence of asthma exacerbations.

Adverse event experienced was limited to purulent hyperemic conjunctivitis successfully treated with betamethasone and chloramphenicol combinated ointment ( 3 applications/die) and pimecrolimus cream (2 applications/die). Only one mild cutaneous exacerbation was observed, successfully treated with topical corticosteroids.

Atopic dermatitis and asthma often occur simultaneously in patients with atopic diathesis. ${ }^{3}$ They are both chronic Th2-driven inflammatory diseases, with an increased expression of IL-4 and IL-13 promoting nitric oxide synthesis, basophils and mast cells activation, eosinophils recruitment to the airways, airway goblet cells development, mucus secretion, and IgE production. ${ }^{4,5}$

Antagonizing IL-4 and IL-4/IL-13 receptor complexes, dupilumab disrupts downstream signaling of the JAK/STAT pathway involved in inflammatory process. ${ }^{6}$ Nowadays it is indicated for the treatment of difficult-to-threat atopic dermatitis in adult patients and also for the treatment of asthma, but only as an additional maintenance therapy. Our real world experience puts in evidence how dupilumab monotherapy should be taken in consideration in patients contemporaneously affected by severe atopic dermatitis and asthma. It could be effective for both of these diseases, minimizing adverse events, and increasing patients compliance.

\section{CONFLICT OF INTEREST}

The authors declare no conflicts of interest.

\section{AUTHOR CONTRIBUTIONS}

$\mathrm{CP}, \mathrm{NS}, \mathrm{ET}$, and AS conceived of the present letter. ET wrote the manuscript. IP, NB, and AM contributed to the final version of the manuscript and supervised the project. VB, PM, AM, SM, and SV contributed to the manuscript revisions. All authors discussed the results and contributed to the final manuscript.
Ersilia Tolino ${ }^{1}$ (D) Ilaria Proietti ${ }^{1}$ (D) Antonella Sarni ${ }^{2}$
Nicoletta Bernardini ${ }^{1}$ (D)

Alessandra Mambrin ${ }^{1}$

Veronica Balduzzi ${ }^{1}$

Patrizia Maddalena ${ }^{1}$

Anna Marchesiello ${ }^{1}$ (D)

Simone Michelini ${ }^{1}$ (iD

Salvatore Volpe ${ }^{1}$ (iD

Nevena Skroza ${ }^{1}$ iD

Concetta Potenza ${ }^{1}$

${ }^{1}$ Dermatology Unit “D. Innocenzi”, Polo Pontino, Sapienza University of

Rome, Rome, Italy

${ }^{2}$ Pneumology UOSD, Department of Medicine, S.M. Goretti Hospital in Latina, Latina, Italy

Correspondence

Ersilia Tolino, Dermatology Unit “D. Innocenzi,” Polo Pontino, Sapienza University of Rome, A. Fiorini Hospital, Via Firenze

1, 04019, Terracina, Italy. Email: ersiliatolino@gmail.com

\section{ORCID}

Ersilia Tolino (D) https://orcid.org/0000-0001-7861-9338

Ilaria Proietti (D) https://orcid.org/0000-0003-3795-3190

Nicoletta Bernardini (D) https://orcid.org/0000-0002-6295-3574

Anna Marchesiello (D) https://orcid.org/0000-0002-5863-4829

Simone Michelini (D) https://orcid.org/0000-0002-3374-7384

Salvatore Volpe (D) https://orcid.org/0000-0002-6367-2344

Nevena Skroza (D) https://orcid.org/0000-0003-4478-5404

\section{REFERENCES}

1. Deeks ED. Dupilumab: a review in moderate to severe asthma. Drugs. 2019;79:1885-1895. https://doi.org/10.1007/s40265-019-01221-x.

2. https://www.ema.europa.eu/en/documents/product-information/dupi xent-epar-product-information_it.pdf

3. Zayed $\mathrm{Y}$, Kheiri B, Banifadel M, et al. Dupilumab safety and efficacy in uncontrolled asthma: a systematic review and meta-analysis of randomized clinical trials. J Asthma. 2018;56(10):1110-1119.

4. Vangipuram R, Tyring SK. Dupilumab for moderate-to-severe atopic dermatitis. Skin Therapy Lett. 2017;22(6):1-4.

5. Corren J. New targeted therapies for uncontrolled asthma. J Allergy Clin Immunol Pract. 2019;7(5):1394-1403. Diamont Z, Dahlen S-E. Type 2 in ammation and the evolving profile of uncontrolled persistent asthma. Eur Med J. 2018;3(4):24-33.

6. Seegraber M, Srour J, Walter A, et al. Dupilumab for treatment of atopic dermatitis. Expert Rev Clin Pharmacol. 2018;11(5):467-474. 